\title{
PROBLEMAS DE COMPORTAMENTO EXTERIORIZADO E AS RELAÇÕES FAMILIARES: REVISÃO DE LITERATURA ${ }^{1}$
}

\author{
Adriane Corrêa Szelbracikowski \\ Maria Auxiliadora Dessen"
}

\begin{abstract}
RESUMO. Pesquisadores têm estudado as relações familiares de crianças com problemas de comportamento exteriorizado, sobretudo, por suas implicações no desenvolvimento humano, ao longo do curso de vida. Os fatores de risco que contribuem para o surgimento e manutenção desses problemas estão relacionados às características da criança, aos processos familiares e às influências dos pares, comunidades e escolas. Neste artigo, discutimos, brevemente, a influência das relações parentais e conjugais em famílias de crianças pré-escolares com problemas de comportamento exteriorizado. No primeiro caso, ênfase é dada às práticas parentais e ao nível de estresse parental; no que tange às relações conjugais, destaque é dado aos padrões de interação em famílias casadas e recasadas e suas possíveis implicações nas relações parentais. Por fim, tecemos algumas considerações a respeito do papel da cultura no desenvolvimento de problemas de comportamento exteriorizado e da necessidade de investigar este tipo de problema sob a perspectiva do desenvolvimento humano.
\end{abstract}

Palavras-chave: relações parentais, relações conjugais, problemas de comportamento exteriorizado.

\section{EXTERNALIZED BEHAVIOR PROBLEMS AND FAMILY RELATIONSHIPS: A REVIEW OF THE LITERATURE}

\begin{abstract}
Researchers have been studying the family relationships of children with externalizing behavior problems owing to the latter's implications on human development during their lives. Risk factors that contribute towards the rise and establishment of such behaviors are associated to children's personal characteristics, family processes, the influence of peers, communities, and schools. The influence of parental and marital relationships in families of children with externalizing behavior problems is discussed. Parental practices and level of parental stress are first emphasized; second, the patterns of interaction in married and re-married families and its implications on parental relationships are highlighted. Finally, the role of the culture in the development of externalizing behavior problems and the necessity of investigating these problems from the human development perspective are debated.
\end{abstract}

Key words: Parental relationships, marital relationships, externalizing behavior problems.

\section{PROBLEMAS DE COMPORTAMIENTO EXTERIORIZADO Y LAS RELACIONES FAMILIARES: REVISIÓN DE LITERATURA}

RESUMEN. Investigadores han estudiado las relaciones familiares de niños con problemas de comportamiento exteriorizado, sobretodo por sus implicaciones en el desarrollo humano, a lo largo del curso de vida. Los factores de riesgo que contribuyen para el surgimiento y manutención de esos problemas están relacionados a las características del niño, a los procesos familiares y a las influencias de los pares, comunidades y escuelas. En este artículo, discutimos, brevemente, la influencia de las relaciones parentales y conyugales en familias de niños preescolares con problemas de comportamiento exteriorizado. En el primer caso, el énfasis es dado a las prácticas parentales y al nivel de estrés parental; en lo que toca a las relaciones conyugales, es dado destaque a los modelos de interacción en familias casadas y re-casadas y sus posibles implicaciones en las relaciones parentales. Por fin, presentamos algunas consideraciones respecto al rol de la cultura en el desarrollo de problemas de comportamiento exteriorizado y de la necesidad de investigar este tipo de problema bajo la perspectiva del desarrollo humano.

Palabras-clave: relaciones parentales, relaciones conyugales, problemas de comportamiento exteriorizado.

\footnotetext{
Apoio: CAPES e CNPq.

* Mestre em Psicologia. Doutoranda pelo Programa de Pós-graduação em Processos de Desenvolvimento Humano e Saúde, Instituto de Psicologia, Universidade de Brasília.

\# Docente do Instituto de Psicologia da Universidade de Brasília. Pós-doutorado pela Universidade de Lancaster, UK e pelo Instituto Max Planck para o Desenvolvimento Humano e Educação, Alemanha.
} 
Atualmente, os limites da família são definidos pelos laços de afetividade e intimidade que as pessoas mantêm umas com as outras, e não apenas pelo parentesco por consangüinidade ou pelo sistema legal que rege as relações familiares (Hodkin, Vacheresse \& Buffett, 1996). Isto significa que a família não é mais vista apenas como um sistema nuclear composto por pai, mãe e seus filhos biológicos, mas incorpora também outras pessoas que fazem parte de sua rede de relações e com quem há afinidades. Considerar a família como unidade de análise significa levar em consideração o desenvolvimento das relações entre os subsistemas, de acordo com os princípios básicos da "teoria sistêmica da família" (Minuchin, 1985, 1988). Nesta teoria, os subsistemas marital, parental e fraternal são interdependentes e compostos por relações diádicas, triádicas e poliádicas (Dessen, 1997; Kreppner, 2001).

A família sofre modificações na sua dinâmica ao longo do tempo, passando por períodos de transição que podem ser normativos e não-normativos, isto é, períodos marcados pela manutenção de padrões e regras que são funcionais e disfuncionais, respectivamente (Kreppner, 1992). As transições normativas propiciam o desenvolvimento de novas formas de interação, regulando os padrões de comunicação de acordo com as mudanças de interesses dos membros da família. Já nas transições não normativas, as circunstâncias estressoras - como insatisfação marital, separação, divórcio, alcoolismo dos pais, dentre outras - exercem um impacto deletério sobre o funcionamento familiar. São as transições familiares não normativas, bem como os fatores biológicos e sociais e as práticas parentais, que influenciam a origem, a manutenção e a evolução dos problemas de comportamento, incluindo os exteriorizados $^{2}$ (Tremblay, 2000). Portanto, para compreendermos os problemas de comportamento, precisamos considerar a influência das relações familiares.

Neste artigo, apresentamos um panorama da dinâmica das relações em famílias de crianças préescolares com problemas de comportamento exteriorizado, destacando a qualidade das relações parentais, os valores e as práticas de socialização e o estresse parental decorrente do relacionamento com os filhos. Os padrões de interação conjugal em famílias

2 Preferimos usar as palavras 'exteriorização' e 'interiorização' traduzidas, respectivamente, do inglês 'externalization' e 'internalization', e não 'externalização' e 'internalização', por constituir um neologismo, sem paradigmas na língua portuguesa. casadas e recasadas e suas possíveis implicações no desenvolvimento de problemas de comportamento exteriorizado são brevemente discutidos. Por fim, tecemos algumas considerações a respeito do papel da cultura nos problemas de comportamento exteriorizado.

\section{RELAÇÕES PARENTAIS EM FAMÍLIAS DE CRIANÇAS PRÉ-ESCOLARES COM PROBLEMAS DE COMPORTAMENTO EXTERIORIZADO}

Comportamentos de descumprimento de regras sociais e de problemas de conduta, tais como agressão física e verbal, roubo, mentira, rebeldia, delinqüência, crueldade física e atos criminosos, estão inseridos na categoria de comportamentos exteriorizados (Rothbaum \& Weisz, 1994; Van der Valk, Verhulst, Stroet \& Boomsma, 1998). Também são incluídos na classe desses comportamentos os padrões de pensamento e sentimento, entre os quais atribuições hostis e irritabilidade. Segundo Hann e Borek (2001), a noção de comportamento exteriorizado como um sistema organizado de comportamentos (respostas) que emergem, intensificam e mudam em conteúdo e freqüência durante o curso do desenvolvimento, em contraposição à noção de atos isolados ou estáticos, é recente.

Dos comportamentos pertencentes a este sistema organizado tem se destacado o comportamento agressivo, por suscitar o interesse de pesquisadores, particularmente daqueles da área de desenvolvimento humano (Szelbracikowski \& Dessen, 2005). Nesta perspectiva, a agressão é vista ao longo do curso de vida do ser humano, com objetivos específicos e formas próprias de cada fase do desenvolvimento, ou seja, nos primeiros anos de vida, nos anos pré-escolares, escolares, adolescência e vida adulta.

Há uma tendência em considerar os problemas de comportamento exteriorizado, incluindo a agressão, como determinados por múltiplos fatores que interagem entre si (Cavell, 2000; Chen \& Muller, 2002). Pesquisas cujo objetivo é examinar os contextos socioculturais que podem expor a criança à situação de risco têm investigado, sobretudo, fatores como ambientes familiares adversos, práticas parentais inadequadas e rejeição de companheiros na escola (Ackerman, Brown \& Izard, 2003; Baker \& Heller, 1996; Brannigan, Gemmell, Peval \& Wade, 2002; Hann \& Borek, 2001; Kim, Hetherington \& Reiss, 1999). Dentre tais fatores, as práticas parentais constituem fortes evidências da associação com problemas de comportamento (Schoppe, Mangelsdorf \& Frosch, 2001). 


\section{COMO SÃO AS PRÁTICAS PARENTAIS NESTAS FAMÍLIAS?}

As práticas parentais em famílias de crianças com problemas de comportamento são caracterizadas, em sua maioria, por coerção, disciplina inconsistente, hostilidade, indiferença, negatividade, restrição emocional, pouca afetividade e apoio, punição e abuso parental (Edens, Cavell \& Hughes, 1999). O uso de punição como prática educativa, além de não resolver os problemas de comportamento, leva a ressentimentos e a dificuldades na interação entre genitores e filhos (Weber, 2005). Segundo Bolsoni-Silva e Marturano (2002), esta prática compromete, muitas vezes, o relacionamento de amizade e cooperação que deveria existir entre pais e filhos, propiciando o surgimento de problemas na infância e na vida adulta, tais como baixa auto-estima, baixa autoconfiança e pouca flexibilidade comportamental frente às dificuldades encontradas no cotidiano.

Em um estudo com famílias de crianças com problemas de comportamento exteriorizado, Dessen e Szelbracikowski (2004) confirmam esses dados sobre as práticas parentais no contexto brasileiro, mas também apontam o uso concomitante de estratégias positivas, de acordo com a percepção dos pais. Neste estudo, os pais relataram que usavam, para os comportamentos inadequados das crianças, a "punição verbal" (ex.: bronca, chamar à atenção) e física (ex.: surras, tapas, chineladas, puxões de orelha), embora o "castigo" (ex.: colocar no quarto, ficar sentado na cadeira, impedir de brincar) e o diálogo (ex.: conversa, conselhos, orientação) fossem também utilizados. Por outro lado, eles demonstravam "contentamento e satisfação" (ex.: elogios, beijos e abraços, alegria, incentivo) diante dos comportamentos adequados. As autoras verificaram, também, que havia pais "indiferentes" aos comportamentos da criança, isto é "não faziam nada" quando ela se comportava de maneira adequada ou inadequada.

Eddy, Leve e Fagot (2001) investigaram se o comportamento agressivo de crianças se desenvolve em famílias cujos pais usam a coerção como principal modo de controle de seus filhos. Eles concluíram, por meio de observações e outras medidas, que os processos de coerção estão associados aos comportamentos agressivos e que são aplicados de forma similar, tanto para meninos quanto para meninas. O processo de coerção é composto por intrusão aversiva de um dos membros da família na atividade da criança; contra-ataque da criança; reforçamento negativo, que aumenta a probabilidade de respostas aversivas futuras da criança; e reforçamento positivo do seu comportamento agressivo. Há, portanto, um efeito recíproco entre a prática parental e o comportamento anti-social, e tais ligações recíprocas podem tornar o comportamento anti-social resistente à mudança, a menos que os fatores que o mantenham sejam modificados (Vuchinich, Bank \& Patterson, 1992).

As práticas parentais dos pais em relação aos seus filhos são cruciais à promoção de comportamentos socialmente adequados; porém, as famílias freqüentemente estimulam comportamentos inadequados por meio de disciplina inconsistente, pouca interação positiva, pouco monitoramento e supervisão insuficiente das atividades (Bolsoni-Silva \& Marturano, 2002; Oliveira \& cols., 2002). Essas práticas disciplinares inadequadas, bem como expressões de raiva dos genitores, podem promover, de forma não intencional, o comportamento anti-social de suas crianças. Tais comportamentos impulsivos são vistos como produtores de efeitos danosos à autoregulação das emoções e, também, como reforçamento de comportamentos agressivos e disruptivos (Kim \& cols., 1999; Wu \& cols., 2002). Assim, as crianças que apresentam comportamentos anti-sociais, nos primeiros anos escolares, incluindo a agressividade, aprenderam a emitir tais comportamentos no contexto familiar (Edens \& cols., 1999).

Há evidências, embora não conclusivas, de que o comportamento e as práticas parentais dos pais exercem uma influência mais poderosa do que a das mães, no que se refere ao desenvolvimento de problemas de comportamento exteriorizado, principalmente em se tratando de meninos (DeKlyen, Biernbaum, Speltz \& Greenberg, 1998). Por exemplo, pais com práticas parentais inadequadas exercem um efeito tanto direto quanto indireto no comportamento anti-social dos meninos e somente um efeito indireto no comportamento de meninas (Kim \& cols., 1999).

As práticas parentais podem ser autoritárias ou exercidas com autoridade. As práticas autoritárias são controladoras e os genitores usam o poder proibitivo e estratégias punitivas visando à obediência absoluta da criança; já as práticas com autoridade, que consistem em respostas apropriadas às demandas e em controle adequado, facilitam o desenvolvimento do comportamento competente da criança (Chen, Dong \& Zhou, 1997; Wu \& cols., 2002). Essas práticas são exercidas de modo individualizado ou de modo compartilhado entre os membros familiares. Por exemplo, no estudo com famílias de crianças com problemas de comportamento exteriorizado realizado por Dessen e Szelbracikowski (2004), a autoridade, na maioria das famílias, era compartilhada por díades constituídas pela mãe e pelo pai/padrasto; e nas 
famílias em que a autoridade era individualizada, esta era exercida mais freqüentemente pela mãe.

Os pais com autoridade, ao interagirem com seus filhos, são mais responsivos e adequados em suas demandas, e ao mesmo tempo mantêm uma disciplina consistente nas suas relações parentais (Muller, Cowan, Cowan, Hetherington \& Clingempeel, 1993; Weber, 2005). Segundo Muller e cols. (1993), esses pais com autoridade, incluindo suporte e controle, têm filhos mais competentes, autoconfiantes e não agressivos do que aqueles que são, ao mesmo tempo, autoritários e permissivos. Na China, as práticas parentais autoritárias e com autoridade influenciam de forma diferenciada o êxito acadêmico e social das crianças. De acordo com Chen e cols. (1997), a prática parental autoritária está associada positivamente à agressão e negativamente à aceitação dos pares, à competência social, à distinção do êxito acadêmico escolar e à distinção de estudante destaque; e a prática parental com autoridade está associada positivamente aos índices de ajustamento social e escolar e negativamente aos problemas de ajustamento.

Não obstante, a eficácia em disciplinar e o uso da punição não são os únicos fatores das relações parentais que podem influenciar o comportamento agressivo da criança; há outros, como a rejeição à criança, o nível de interesse e o envolvimento positivo dos pais, a habilidade em monitorar o paradeiro dos filhos e o nível de estabilidade e organização que os pais criam no ambiente familiar (Szelbracikowski \& Dessen, 2005). A dificuldade em desempenhar a parentalidade pode tornar a família um caos, particularmente quando os pais não conseguem fazer distinção entre os seus papéis na família e os papéis das crianças (Cavell, 2000; Coie \& Dodge, 1998). Essas dificuldades na socialização dos filhos podem se tornar fonte de estresse para os genitores, influenciando o surgimento e a manutenção dos problemas de comportamento exteriorizado.

\section{QUAL O NÍVEL DE ESTRESSE PARENTAL NESTAS FAMÍLIAS?}

O estresse parental influencia e é influenciado pelas práticas disciplinares empregadas pelos pais, pelos problemas econômicos enfrentados pela família, pela perda de emprego e pelos tipos de família e seus padrões específicos de funcionamento (Dessen \& Braz, 2005a, 2005b). O estresse constitui um dos fatores responsáveis pelo aumento da irritabilidade dos genitores, da probabilidade de os pais iniciarem ou manterem trocas aversivas com seus filhos ou contra- atacarem em resposta à agressividade da criança (Kazdin \& Whitley, 2003).

Dessen e Szelbracikowski (2004) aplicaram uma escala de estresse parental (Abidin, 1995) aos pais, aos padrastos e às mães de crianças pré-escolares com problemas de comportamento exteriorizado. Elas encontraram pouca diferença entre pais e mães nas subescalas "sofrimento parental" e "interações disfuncionais". A primeira subescala diz respeito ao sofrimento e angústia que os genitores experienciam no seu papel de pai/mãe; a segunda, avalia as percepções que os genitores têm de seus filhos que não são compatíveis com as suas expectativas e as percepções de suas interações com a criança que não reforçam o seu papel de pai/mãe. Mas, na subescala "criança difícil", que mede algumas características comportamentais básicas das crianças que as tornam fáceis ou difíceis de manejar, não houve diferença entre pais e mães. No entanto, os padrastos apresentaram um índice mais elevado de estresse parental que os pais e as mães, nas três subescalas (sofrimento parental, interação disfuncional e criança difícil), indicando que em famílias recasadas o estresse foi mais elevado. Este dado precisa ser mais bem investigado em estudos com amostras significativas da população brasileira.

As origens e a manutenção do estresse parental são multivariadas e dependem do acesso e da disponibilidade de recursos percebidos pelos genitores nas demandas da parentalidade, da percepção dos pais sobre o comportamento das crianças e da sensação de competência parental (Deater-Deckard \& Scarr, 1996). De acordo com esses autores, o estresse parental está fortemente associado a relações de apego inseguras, ao abuso e à parentalidade autoritária e rude, bem como à pobreza, à depressão materna e a crianças hiperativas, com doenças crônicas e autismo severo.

Há outros aspectos das relações parentais, além do estresse, que podem colocar as crianças em situações de risco quanto ao surgimento de problemas emocionais e de comportamento, dentre os quais os conflitos familiares. Jenkins e Smith (1991) realizaram um estudo com 119 famílias com crianças entre 9 e 12 anos de idade e verificaram que o conflito parental é deletério para a criança, evidenciando pouco monitoramento e cuidado parental reduzido, bem como níveis elevados de agressão física às crianças.

Os conflitos familiares presentes, particularmente, na relação conjugal constituem outra fonte de estresse em famílias de crianças com problemas de comportamento exteriorizado, influenciando as relações parentais e, conseqüentemente, a manutenção e evolução dos problemas de comportamento 
exteriorizado (Dessen \& Szelbracikowski, 2004), conforme discutido a seguir.

\section{RELAÇÕES CONJUGAIS EM FAMÍLIAS DE CRIANÇAS PRÉ-ESCOLARES COM PROBLEMAS DE COMPORTAMENTO EXTERIORIZADO}

Existem fortes evidências dos prejuízos causados pela disfuncionalidade familiar no desenvolvimento dos filhos e da existência de uma correlação positiva entre dificuldades e problemas de comportamento da criança e os distúrbios na relação conjugal (Emery, 1982; Gottman, 1998; Kreppner \& Ullrich, 1998). O divórcio é considerado um evento estressor, pois modifica a estrutura familiar, alterando a dinâmica das relações familiares e, conseqüentemente, o ajustamento das crianças (Malone \& cols., 2003).

Malone e cols. (2003) realizaram um estudo para verificar os efeitos do divórcio de genitores no ajustamento de crianças com problemas de comportamento exteriorizado que freqüentavam da $1^{\mathrm{a}}$ à $8^{a}$ séries quando seus pais se divorciaram. Eles concluíram que as meninas de $1^{\mathrm{a}}$ a $5^{\mathrm{a}}$ série não foram afetadas pela experiência do divórcio dos pais, ao contrário dos meninos, que apresentaram um aumento dos problemas de comportamento exteriorizado, no ano anterior ao divórcio. Este aumento persistiu até um ano após o divórcio. Os meninos que freqüentavam da $6^{\mathrm{a}}$ à $8^{\mathrm{a}}$ séries também apresentaram um aumento de problemas de comportamento exteriorizado no ano anterior ao divórcio, mas um decréscimo no ano do divórcio, que persistiu nos anos seguintes. Portanto, as relações maritais infelizes e o divórcio ou a separação dos cônjuges aumentam o risco do surgimento de psicopatologias ou problemas de ajustamento (Erel \& Burman, 1995).

Considerando-se haver uma correlação consistente e bem-fundamentada entre a qualidade da relação conjugal e a qualidade da relação dos genitores com seus filhos e, por consequiência, com o desenvolvimento e o comportamento infantil, o estudo dos processos de desenvolvimento, qualquer que seja a sua dimensão, deve abranger a compreensão das relações conjugais e parentais (Belsky,1984; Dessen \& Braz, 2005b; Emery, 1982; Erel \& Burman, 1995; Gottman, 1998).

\section{AS RELAÇÕES PARENTAIS E CONJUGAIS EM FAMÍLIAS CASADAS E RECASADAS DIFEREM?}

Os padrões de interação e relação de crianças e adolescentes com problemas de comportamento exteriorizado variam de acordo com o tipo de estrutura familiar, isto é, se eles vivem com as famílias biológicas ou pertencem a famílias recasadas (Kim \& cols., 1999). Atualmente, há um número cada vez mais crescente de famílias recasadas, compostas por um genitor biológico e a madrasta ou padrasto (Kaloustian, 1998) e de famílias alternativas (Stratton, 2003).

A dinâmica das famílias casadas e recasadas apresenta características distintas, particularmente no que se refere às relações parentais e aos papéis do padrasto e da madrasta. Dessen e Szelbracikowski (2004) verificaram em seu estudo que, na maioria das famílias constituídas por mãe, criança e padrasto, as mães e os padrastos percebiam que a autoridade era compartilhada pela díade mãe-padrasto; que as estratégias de socialização utilizadas eram a "punição física" e a "punição verbal"; e que as mães eram as principais responsáveis pelos cuidados dos filhos e afazeres da casa, com a participação do padrasto apenas na atividade de fazer compras. O envolvimento do padrasto na vida familiar era parcial; as interações das crianças dessas famílias com parentes e vizinhos eram positivas, mas com o padrasto eram conflituosas e distantes.

Kim e cols. (1999) investigaram a influência das relações de genitores e padrastos sobre adolescentes com problemas de comportamento exteriorizado, de ambos os sexos, provenientes de famílias casadas e recasadas. Os resultados indicaram que comportamentos hostis e aversivos de padrastos são preditores mais relevantes de problemas de comportamento que de pais biológicos, sugerindo que a figura do padrasto tem um potencial maior para desencadear problemas de ajustamento na criança. Os padrastos tendem, também, a se manter mais distantes e mostrar-se menos envolvidos e menos controladores que os pais biológicos. Por outro lado, os pais biológicos, talvez devido ao seu maior envolvimento com a criança, estão mais propensos a criticar e a entrar em atrito com ela sobre questões menores e sem importância.

Em se tratando de relacionamento conjugal, independentemente do tipo de família, há consenso na literatura de que os conflitos conjugais podem trazer prejuízos não só para os cônjuges, mas também para o desenvolvimento dos filhos (Erel \& Burman, 1995). Um desses conflitos refere-se à co-parentalidade, que é entendida como a cooperação entre ambos os genitores na criação e educação de seus filhos. Schoppe e cols. (2001) mostram que as interações familiares negativas, isto é, com co-parentalidade enfraquecida, afeto negativo e estrutura familiar pouco 
adaptativa, foram fortemente associadas às crianças que apresentavam problemas de comportamento exteriorizado. De todos esses aspectos, a coparentalidade foi o fator preponderante.

Em suma, devido à falta de laços biológicos e de uma história familiar conjunta, verifica-se que há uma ambigüidade nos papéis de responsabilidade de padrastos e madrastas, ocorrendo, freqüentemente, certa resistência e problemas de comportamento da criança (enteada), especialmente no início do recasamento (Kim \& cols., 1999). Há, também, evidências empíricas de que os problemas de comportamento exteriorizado são mais freqüentes em crianças cujos pais são divorciados e recasados do que em crianças cujos pais não são divorciados (Bray \& Berger, 1993; Hetherington, 1993). No entanto, alguns estudos têm encontrado pouca diferença no ajustamento das crianças de famílias divorciadas e recasadas (Cherlin \& Furstenberg, 1994), sugerindo a necessidade de intensificar as pesquisas para obter dados mais conclusivos.

\section{CONSIDERAÇÕES FINAIS: CULTURA E PROBLEMAS DE COMPORTAMENTO EXTERIORIZADO}

A influência dos genitores no desenvolvimento das crianças deve ser compreendida considerando as interrelações dos contextos, das características e do papel ativo da criança em desenvolvimento, todos em constantes mudanças. Apesar de o desenvolvimento infantil variar de acordo com os valores e crenças de uma cultura ou de uma subcultura, há um número limitado de pesquisas sobre as diferenças culturais nos processos familiares relacionados aos problemas de comportamento exteriorizado (Hann \& Borek, 2001).

As relações familiares envolvem a transmissão de significado e cultura de uma geração à seguinte, estabelecendo um padrão comum de valores e normas (Kreppner, 2000). A família e a sua rede de relações pode ser considerada como uma instituição responsável pela proteção do funcionamento biológico, pela sobrevivência e pela manutenção e transmissão de valores culturais, tradições e significados, e esta transmissão se dá, sobretudo, nas relações parentais (Valsiner, Branco \& Dantas, 1997).

Os valores e as crenças parentais constituem o principal ponto de contato entre a cultura social mais ampla e a cultura pessoal, exercendo influência nas práticas dos genitores dirigidas às suas crianças (Valsiner \& cols., 1997). Os genitores geralmente têm sua própria subcultura familiar, objetivos pessoais e valores na criação dos seus filhos, de modo que algumas crianças são encorajadas a se envolver em determinados tipos de atividade e desencorajadas em relação a outros. Quando a criança, no seu processo de desenvolvimento, é exposta à agressividade, seja na forma de punição ou de violência dos pais ou familiares, pode ocorrer o desenvolvimento de sentimentos de hostilidade e desconfiança e percepções negativas em relação aos outros, particularmente no relacionamento com pares e professores, na escola, e com pais e irmãos, no contexto familiar (Hann \& Borek, 2001).

Destarte, faz-se necessário compreender os comportamentos exteriorizados em uma perspectiva cultural, particularmente a agressão, uma vez que esta é um produto da subcultura familiar e da cultura em geral (ver, por exemplo, Szelbracikowski \& Dessen, 2005, Tremblay, 2000). Ênfase deve ser dada às origens e às trajetórias dos problemas de comportamento exteriorizado em crianças pré-escolares, levando em consideração a estrutura familiar e os padrões de relações conjugais e parentais que caracterizam a cultura das famílias.

Os estudiosos, embora tenham unido esforços para compreender como ocorrem, por exemplo, os processos de socialização da criança em diferentes ambientes, têm negligenciado aspectos como a hereditariedade e características do grupo de pares dos indivíduos em desenvolvimento, supervalorizando dados correlacionais e falhando ao interpretar, especialmente, os efeitos da hereditariedade (Collins, Maccoby, Steinberg, Hetherington \& Bornstein, 2000). Os estudos sobre as relações familiares podem constituir uma alternativa valiosa para ajudar a responder a questões polêmicas sobre as influências de fatores herdados e adquiridos no que tange à origem e evolução de comportamentos exteriorizados.

A família tem a função de transmitir não somente genes, mas também significado e cultura. Cada família, com sua maneira particular de manter a motivação e o significado entre seus membros, pode ser interpretada como representativa de uma cultura particular, um tipo de unidade que produz modos comuns de comunicar-se com o mundo externo e avaliar suas experiências. Nesta perspectiva, a família é a principal via de transmissão de significados e cultura para a criança. A Psicologia, no Brasil, precisa investir mais em estudos que investiguem os fatores ambientais e genéticos, uma vez que os padrões das interações e relações familiares das crianças brasileiras são bastante distintos (Dessen \& Torres, 2002) e podem ser as principais fontes de risco para o desenvolvimento de problemas de comportamento exteriorizado. A implementação de pesquisas desta 
natureza contribuiria para subsidiar, também, as práticas clínica e escolar, com implicações em programas preventivos sobre a delinqüência nas fases subsequientes ao período pré-escolar.

\section{REFERÊNCIAS}

Abidin, R. R. (1995). Parenting stress index. Odessa: Psychological Assessement Resources.

Ackerman, B., Brown, E. \& Izard, C. (2003). Continuity and change in levels of externalizing behavior in school of children from economically disadvanteged families. Child Development, 74, 694-709.

Baker, B. L. \& Heller, T. L. (1996). Preschool children with externalizing behaviors: Experience of fathers and mothers. Journal of Abnormal Child Psychology, 24, 513-532.

Belsky, J. (1984). The determinants of parenting: A process model. Child Development, 55, 83-96.

Bolsoni-Silva, A. T. \& Marturano, E. M. (2002). Práticas educativas e problemas de comportamento: uma análise à luz das habilidades sociais. Estudos de Psicologia, 7, 227-235.

Brannigan, A., Gemmell, W., Peval, D. J. \& Wade, T. J. (2002). Self-control and social childhood misconduct and aggression: The role of family structure, hyperactivity and hostile parenting. Canadian Journal of Criminology, 44, 119-142.

Bray, J. H. \& Berger, H. (1993). Developmental issues in stepfamilies research project. Family relationships and parentchild interactions. Journal of Family Psychology, 7, 76-90.

Cavell, T. A. (2000). Working with parents of aggressive children: A practitioner's guide. Washington: American Psychological Association.

Chen, X., Dong, Q. \& Zhou, H. (1997). Authoritative and authoritarian parenting practices and social and school performance in chinese children. International Journal of Behavioral Development, 21, 855-873.

Chen, X. \& Muller, J. (2002). Aggression and violence in Youth (Introduction). International Society for the Study of Behavioural Development (Newsletter), 42, 1.

Cherlin, A. \& Furstenberg, F. (1994). Stepfamilies in the United States: A reconsideration. Annual Review of Sociology, 20, 359-381.

Coie, J. D. \& Dodge, K. A. (1998). Aggression and antisocial behavior. In W. Damon \& N. Eisenberg (Eds.), Handbook of child psychology: Social, emotional, and personality Development (pp. 779-840). Toronto: Wiley.

Collins, W. A., Maccoby, E. E., Steinberg, L., Hetherington, E. M. \& Bornstein, M. H. (2000). Contemporary research on parenting: The case for nature and nurture. American Psychologist, 55, 218-232.

Deater-Deckard, K. \& Scarr, S. (1996). Parenting stress among dual-earner mothers and fathers: Are there gender differences? Journal of Family Psychology, 10, 45-59.
DeKlyen, M., Biernbaum, M. A., Speltz, M. L. \& Greenberg, M. T. (1998). Fathers and preschool behavior problems. Developmental Psychology, 34, 264-275.

Dessen, M. A. (1997). Desenvolvimento familiar: transição de um sistema triádico para poliádico. Temas em Psicologia, 3, 5161.

Dessen, M. A. \& Braz, M. P. (2005a). A família e suas interrelações com o desenvolvimento humano. Em M. A. Dessen \& A. L. C. Junior (Orgs.), A ciência do desenvolvimento: tendências atuais e perspectivas futuras (pp. 113-131). Porto Alegre: ArtMed.

Dessen, M. A. \& Braz, M. P. (2005b). As relações maritais e sua influência nas relações parentais. Em M. A. Dessen \& A. L. C. Junior (Orgs.), A ciência do desenvolvimento: tendências atuais e perspectivas futuras (pp.132-152). Porto Alegre: ArtMed.

Dessen, M. A. \& Szelbracikowski, A. C. (2004). As crianças préescolares com problemas de comportamento exteriorizado e dinâmica familiar. Interação em Psicologia, 8, 171-180

Dessen, M. A. \& Torres, C. V. (2002). Family and socialization factors in Brazil: An overview. In W. J. Lonner, D. L. Dinnel, S. A. Hayes \& D. N. Sattler (Eds.), OnLine Readings in Psychology and Culture (Unit 13, Chapter 2). Bellingham, Washington, USA: Western Washington University, Center for Coss-Cultural Research. Disponivel em: <htttp://www.wwu.edu/ culture>. (Acesso em 24/11/2003).

Eddy, J. M., Leve, L. D. \& Fagot, B. I. (2001). Coercive family processes: A replication and extension of Patterson's coercion model. Aggressive Behavior, 27, 14-25.

Edens, J., Cavell, T. \& Hughes, J. (1999). The self-systems of aggressive children: A cluster-analytic investigation. Journal of Child Psychology and Psychiatry, 40, 441-453.

Emery, R. E. (1982). Interparental conflict and the children of discord and divorce. Psychological Bulletin, 92, 310-330.

Erel, O. \& Burman, B. (1995). Interrelatedness of marital relations and parent-child relations: A meta-analytic review. Psychological Bulletin, 118, 108-132.

Gottman, J. M. (1998). Psychology and the study of marital processes. Annual Review of Psychology, 49, 169-197.

Hann, D. M. \& Borek, N. (2001). Taking stock of risk factors for child/youth externalizing behavior problems. Washington: National Institute of Mental Health.

Hetherington, E. M. (1993). An overview of the Virginia longitudinal study of divorce and remarriage with a focus on early adolescence. Journal of Family Psychology, 7, 39-56.

Hodkin, B., Vacheresse, A. \& Buffett, S. (1996). Concept of family: Methodological issues in assessing perceived family membership. In M. Cusinato (Ed.), Research on family: Resources and needs across the world (pp. 45-54). Milan: LED-Edicioni Universitarie.

Jenkins, J. M. \& Smith, M. A. (1991). Marital disharmony and children's behaviour problems: Aspects of a poor marriage that affect children adversely. Journal of Child Psychology and Psychiatry and Allied Disciplines, 32, 793-810. 
Kaloustian, S. M. (Org.), (1998). Família brasileira: a base de tudo. São Paulo: Cortez.

Kazdin, A. E. \& Whitley, M. K. (2003). Treatment of parental stress to enhance therapeutic change among children referred for aggressive and antisocial behavior. Journal of Consulting and Clinical Psychology, 71, 504-515.

Kim, J., Hetherington, E. \& Reiss, D. (1999). Associations among family relationships, antisocial peers and adolescents' externalizing behaviors: Gender and family type diferences. Child Development, 70, 1209-1230.

Kreppner, K. (1992). Development in a developing context: Rethinking the family's role for children's development. In L. T. Winegar \& J. Valsiner (Eds.), Children's development within social context (pp. 161-179). Hillsdale: Lawrence Erlbaum.

Kreppner, K. (2000). The child and the family: Interdependence in developmental pathways. Psicologia: Teoria e Pesquisa, 16, 11-22.

Kreppner, K. (2001). Sobre a maneira de produzir dados no estudo da interação social. Psicologia: Teoria e Pesquisa, 17, 97-107.

Kreppner, K. \& Ullrich, M. (1998). Talk to mom and dad, and listen to what is in between. In M. Hofer, P. Noack \& J. Youniss (Eds.), Verbal interaction and development in families with adolescentes (pp. 83-108). Greenwich: Ablex.

Malone, P. S., Lansford, J. E., Castellino, D. R., Berlin, L. J., Dodge, K. A., Bates, J. E. \& Petit, G. S. (2003). Divorce and child behavior problems: Applying latent change score models to life event data. Disponível em: <http://www.duke.edu/ malone/writtings/ip_mal1.htm>. (Acesso em 20/07/2003).

Minuchin, P. (1985). Families and individual development: Provocations from the field of family therapy. Child Development, 56, 289-302.

Minuchin, P. (1988). Relationships within the family: A systems perspectives on development. In R. Hinde \& J. StevensonHinde (Eds.), Relationships within families: Mutual influences (pp. 8-25). Oxford: Clarendon Press \& University Press.

Muller, N. B., Cowan, P. A., Cowan, C. P., Hetherington, E. M. \& Clingempeel, W. G. (1993). Externalizing in preschoolers and early adolescents: A cross-study replication of a family model. Developmental Psychology, 29, 3-18.

Oliveira, E. A., Marin, A. H., Pires, F. B., Frizzo, G. B., Ravanello, T. \& Rossato, C. (2002). Estilos parentais autoritário e democrático-recíproco intergeracionais, conflito conjugal e comportamentos de externalização e internalização. Psicologia: Reflexão e Crítica, 15, 1-11.
Rothbaum, F. \& Weisz, J. R. (1994). Parental caregiving and child externalizing behavior nonclinical samples: A meta-analysis. Psychological Bulletin, 116, 55-74.

Schoppe, S. J., Mangelsdorf, S. C. \& Frosch, C. A. (2001). Coparenting, family process and family structure: Implications for preschoolers' externalizing behavior problems. Journal of Family Psychology, 15, 526-545.

Stratton, P. (2003). Contemporary families as contexts for development. In J. Valsiner \& K. Connolly (Eds.), Handbook of developmental psychology (pp. 333-357). London: Sage.

Szelbracikowski, A. \& Dessen, M. A. (2005). Compreendendo a agressão na perspectiva do desenvolvimento humano. Em M. A. Dessen \& A. L. C. Junior (Orgs.), A ciência do desenvolvimento: tendências atuais e perspectivas futuras (pp. 113-131). Porto Alegre: ArtMed.

Tremblay, R. E. (2000). The development of aggressive behaviour during childhood: What have we learned in the past century? International Journal of Behavioral Development, 24, 129141.

Valsiner, J., Branco, A. U. \& Dantas, C. (1997). Socialization as co-construction: Parental belief orientations and heterogeneity of reflection. In J. E. Grusec \& L. Kuczynski (Eds.), Parenting and children's internalization of values (pp. 283-304). New York: Wiley.

Van der Valk, J. C., Verhulst, F. C., Stroet, T. M. \& Boomsma, D. I. (1998). Quantitative genetic analysis of internalizing and externalizing problems in a large sample of 3-year-old twins. Twin Research, 1, 25-33.

Vuchinich, S., Bank, L. \& Patterson, G. R. (1992). Parenting, peer and the stability of antisocial behavior in preadolescent boys. Developmental Psychology, 28, 510-521.

Weber, L. (2005). Eduque com carinho: para pais e filhos. Curitiba: Juruá.

Wu, P., Robinson, C. C., Yang, C., Hart, C. H., Olsen, S. F., Porter, C. L., Jin, S., Wo, J. \& Wu, X. (2002). Similarities and differences in mother's parenting of preschoolers in China and United States. International Journal of Behavioral Development, 26, 481-491.

Recebido em 08/11/2005 Aceito em 13/03/2006

Endereço para correspondência: Maria Auxiliadora Dessen. Universidade de Brasília-UnB; Instituto de Psicologia/PED; Laboratório de Desenvolvimento Familiar; Campus Universitário Darcy Ribeiro; Brasília-DF; CEP: 70.910-900. E-mail: dessen@unb.br 